\title{
Cambios en la función hepática causados por COVID-19 y su impacto en el resultado clínico del paciente: una revisión sistemática
}

\section{Changes in liver function tests caused by COVID-19 and impact on patient outcome: a systematic review}

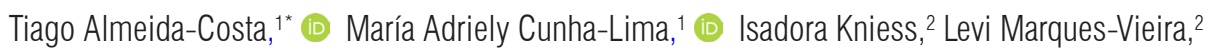

Leda María Delmondes-Freitas Trindade. ${ }^{3}$ (iD)

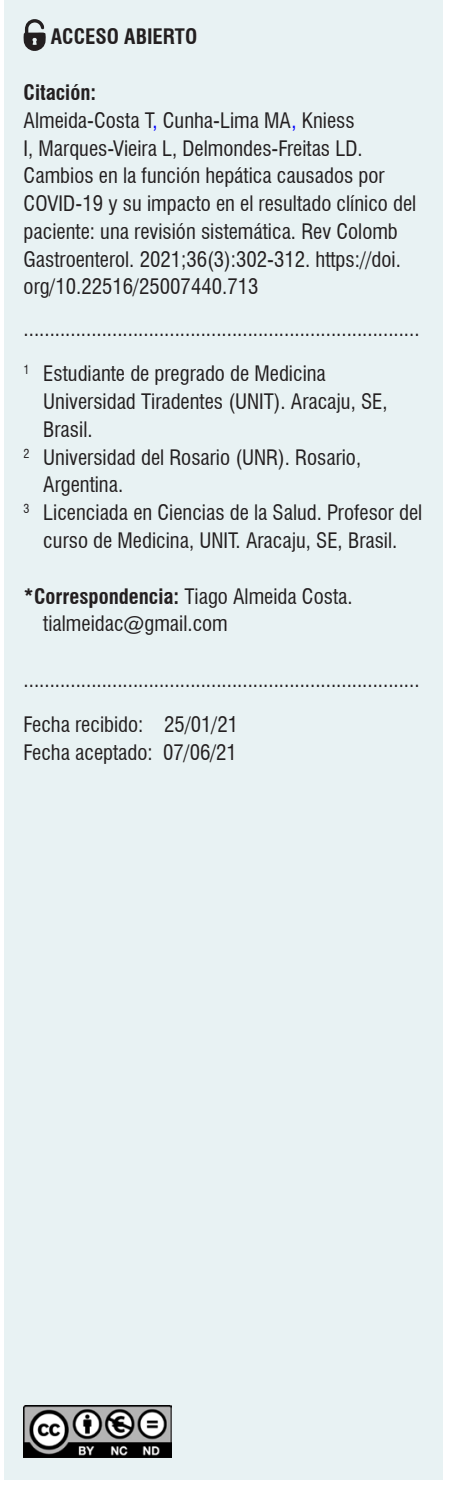

\section{Resumen}

Introducción: En marzo de 2020, la Organización Mundial de la Salud (OMS) decretó la pandemia de la enfermedad por coronavirus de 2019 (COVID-19), que consiste en la infección por coronavirus del síndrome respiratorio agudo grave de tipo 2 (SARS-CoV-2). Este virus utiliza la enzima convertidora de angiotensina II (ECA-II) como receptor celular humano, que está presente en el tejido pulmonar, cardíaco, gastrointestinal, hepático, renal y vascular, lo que configura un potencial de afectación multisistémica por parte del patógeno. El hígado puede resultar dañado tanto por la liberación excesiva de citocinas inflamatorias en COVID-19 como por la adopción de fármacos con potencial hepatotóxico en el tratamiento de sus síntomas. Objetivo: analizar la relación entre los cambios en la función hepática causados por el SARS-CoV-2 y su impacto en el pronóstico del paciente. Métodos: el presente estudio consiste en una revisión sistemática, realizada a partir de estudios seleccionados de las bases de datos PMC, LILACS y SciELO. Después de aplicar los criterios de inclusión y exclusión, se definieron 30 artículos para componer la base de datos de este estudio. Resultados: La enzima aspartato-aminotransferasa (AST) estaba aumentando en mayor prevalencia, con un total de 4695 casos, mientras que la alanina-aminotransferasa (ALT) estaba elevada en 3226 casos. Se observa que los pacientes que presentaban síntomas digestivos tenían más probabilidades de presentar daño hepatocelular y, en consecuencia, alteraciones enzimáticas. Además, la mortalidad ocurrió en el 28,9\% de los casos de pacientes con función hepática alterada, mientras que, en aquellos con función normal, esta tasa fue del 9 \%. Conclusión: es evidente que existe una relación entre la afectación hepática por COVID-19 y su mortalidad. Sin embargo, todavía existe una limitación en la cantidad y, principalmente, en la homogeneidad de los estudios que realizaron dicha valoración.

\section{Palabras clave \\ Pruebas de función hepática, mortalidad, infecciones por coronavirus.}

\section{Abstract}

Introduction: In March 2020, the World Health Organization declared COVID-19, a disease caused by SARSCoV-2 infection, a global pandemic. This virus uses human angiotensin-converting enzyme 2 (ACE2) as its receptor for entry. ACE2 is found in pulmonary, cardiac, gastrointestinal, hepatic, renal and vascular tissues, thus posing a potential risk for multisystemic involvement. The excessive release of inflammatory cytokines in COVID-19, as well as the use of medicines with hepatotoxic potential for the treatment of its symptoms, can damage the liver. Objective: To analyze the relationship between changes in liver function tests caused by SARS-CoV-2 infection and their impact on patient prognosis. Methodology: This is a systematic review of studies selected from the PMC, LILACS and SciELO databases. After applying the inclusion and exclusion criteria, 30 articles were included in the final sample for analysis. Results: Elevated AST (aspartate aminotransferase) enzyme levels were reported most frequently and were found in 4695 cases, while ALT (alanine aminotransferase) elevation was described in 3226 cases. It was observed that patients with digestive symptoms were more likely to present hepatocellular damage and, consequently, enzymatic alterations. Furthermore, $28.9 \%$ of individuals with impaired liver function died, compared to $9 \%$ of patients with normal function. Conclusion: It is evident that there is a relationship between liver involvement in COVID-19 and mortality. However, there is still a limitation in the number and, more importantly, the homogeneity of the research that performed this assessment.

\section{Keywords}

Liver function tests; Mortality; Coronavirus infections. 


\section{INTRODUCCIÓN}

En diciembre de 2019 China informó públicamente sus primeros casos de la enfermedad por coronavirus de 2019 (COVID-19), una enfermedad causada por la infección del coronavirus del síndrome respiratorio agudo grave de tipo 2 (SARS-CoV-2), y en marzo de 2020, debido a la propagación global del virus, la Organización Mundial de la Salud (OMS) decretó oficialmente la pandemia de COVID-19 $9^{(1)}$. El SARS-CoV-2, el nuevo coronavirus, se caracteriza por ser un virus envuelto de cadena simple de ácido ribonucleico (ARN) de la familia Coronaviridae y tiene un período de incubación, alrededor de 14 días, con una mediana de 4 a 6 días $^{(2,3)}$. La transmisión de COVID-19 ocurre de persona a persona por contacto o apretón de manos y por el contacto con gotitas de saliva, estornudos o tos de un individuo infectado emitidas por el aire ${ }^{(3)}$.

El mecanismo de patogenia del SARS-CoV-2 consiste en utilizar la enzima convertidora de angiotensina II (ECA-II) como receptor para su entrada en la célula ${ }^{(2)}$. La ECA-II está presente no solo en los alvéolos pulmonares, sino también en miocitos cardíacos, tracto gastrointestinal, hígado, riñones, células endoteliales vasculares y células musculares en arterias, lo que refleja la afectación de varios órganos y tejidos, y esto justifica la variedad de síntomas de esta enfermedad ${ }^{(4)}$.

La presentación clínica de la COVID-19 es variable y puede ser asintomática, con síntomas leves a moderados, especialmente fiebre, dolor de cabeza, tos, congestión nasal y síntomas gastrointestinales; hasta manifestaciones graves, especialmente en un paciente con asociación de comorbilidades y afectación multisistémica ${ }^{(5,6)}$. La fisiopatología de la COVID-19 aún no se ha dilucidado por completo; sin embargo, uno de sus mecanismos es la liberación exacerbada de citocinas inflamatorias que culmina en afectación multisistémica, que puede desencadenar cambios vasculares y coagulativos ${ }^{(7)}$.

En cuanto al daño hepático, debido al estado de hiperinflamación por la "tormenta de citocinas" y desregulación de la coagulación, hay análisis de laboratorio de trombocitopenia leve, prolongación del tiempo de protrombina, elevación de ferritina y proteína C-reactiva $(\mathrm{PCR})^{(7)}$. Existen reportes en la literatura de daño hepático asociado con sepsis secundaria a la COVID-19 $9^{(8,9)}$, así como una descripción del uso de fármacos hepatotóxicos en el manejo terapéutico sintomático de la COVID-19 y sus complicaciones ${ }^{(4,10)}$.

Para evaluar la fisiología del hígado de forma global e identificar las lesiones hepáticas se utilizan marcadores bioquímicos: aspartato-aminotransferasa (AST), alanina-aminotransferasa (ALT), bilirrubina total y sus fracciones, fosfatasa alcalina y $\gamma$-glutamiltransferasa (GGT), que cuando están por encima de los valores de referencia indican cambios en la función hepática y posible daño hepatocelular ${ }^{(11)}$.
$\mathrm{Al}$ ser aún una enfermedad muy reciente, la COVID-19 carece de más estudios para evaluar su posible daño tisular, una vez que ya se ha reportado el acceso a los distintos órganos por parte del virus. Dado que el objetivo de esta revisión es correlacionar el desarrollo de alteraciones de la función hepática por SARS-CoV-2 y su impacto en la evolución clínica del paciente con la infección, vale la pena enfatizar que hasta ahora no está claro si el mecanismo que causa daño hepático en la infección por SARS-CoV-2 es el resultado de la enfermedad y sus mecanismos fisiopatológicos o el tratamiento actualmente utilizado.

\section{MATERIAL Y MÉTODOS}

El presente estudio consiste en una revisión sistemática que tiene como objetivo, principalmente, correlacionar las alteraciones hepáticas provocadas por la infección por SARSCoV-2 con el pronóstico del paciente con esta infección. Las bases de datos PubMed Central ${ }^{\oplus}$ (PMC), la Literatura Latinoamericana y del Caribe en Ciencias de la Salud (LILACS) y la Biblioteca Electrónica Científica en Línea (SciELO) fueron seleccionadas para investigar el tema relacionado con este estudio. Además, se definieron los siguientes descriptores para realizar la investigación dirigida al tema propuesto: "lesión" (D1), "hepática" (D2), "coronavirus" (D3) y "COVID-19" (D4), además del operador Booleano $\ll Y »$. Ante esto, se utilizaron asociaciones $\ll \mathrm{D} 1 »$.

Luego, se definieron los siguientes filtros para ser aplicados en las búsquedas: ensayo aleatorizado, estudios observacionales (cohortes, transversales y de casos y controles), series de casos, artículos publicados en el último año y texto completo, que se utilizaron como criterios para inclusión en el estudio. Además, como es un tema reciente, no hubo restricción en el idioma. Los criterios de exclusión utilizados fueron estudios que no discutieron el tema o no presentaron datos suficientes para lograr el objetivo propuesto en esta revisión. También se excluyeron editoriales, comentarios, metaanálisis y artículos de revisión. Cabe destacar que, por tratarse de una enfermedad reciente, no fue posible rastrear homogeneidad en todos los artículos seleccionados.

Luego de aplicar los criterios de inclusión y exclusión, se encontraron 4742 artículos en las bases de datos, se excluyeron 4523 y solo se incluyeron 219 artículos. Posteriormente, se leyeron títulos y resúmenes con el fin de excluir estudios que no hablaran de la temática propuesta, por lo que se eligieron 47 trabajos para lectura completa. Luego de la lectura, se excluyeron 17 artículos que no contenían datos suficientes para poder cumplir con los objetivos de este estudio, por lo que se definieron 30 artículos para componer la base de datos para esta revisión sistemática (Figura 1). 


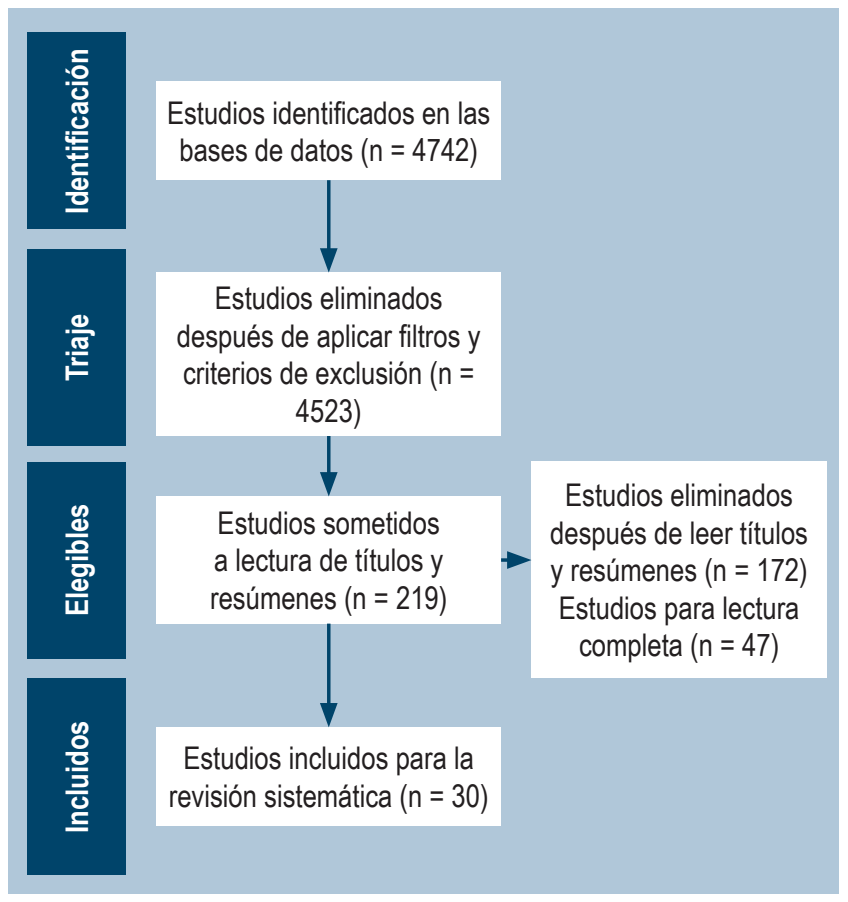

Figura 1. Diagrama de flujo PRISMA del estudio. Datos de la investigación, 2020.

No se seleccionaron artículos de la base de datos LILACS, ya que solo se encontraron 2 artículos durante la búsqueda, los cuales no discutieron el tema propuesto. En SciELO hubo 4 artículos en la investigación, de los cuales solo 1 tenía datos suficientes para lograr el objetivo propuesto en este estudio. Cabe señalar que todo el proceso de selección de artículos se llevó a cabo de forma independiente por dos revisores.

Para aplicar el índice kappa para analizar el índice de concordancia entre los revisores, la selección de los artículos se realizó de forma independiente por los autores María Adriely Cunha Lima (ML) y Tiago Costa (TC), y si el valor determinaba, por ejemplo, falta de concordancia, concordancia pobre o leve, se elegiría un tercer revisor (Isadora Kniess [IK]). Por tanto, IK elegiría un artículo en el punto de intersección de los dos revisores anteriores para eliminar la discrepancia inicial (Tabla 1).

Tabla 1. Índice de contingencia

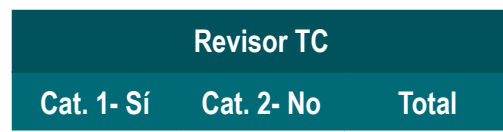

\begin{tabular}{|c|c|ccc|}
\hline \multirow{2}{*}{ Revisor ML } & Cat. 1- Sí & 28 & 13 & 41 \\
\cline { 2 - 5 } & Cat. 2- No & 17 & 4684 & 4701 \\
\hline & Total & 45 & 4697 & 4742 \\
\hline
\end{tabular}

Cat.: categoría. Fuente: datos de la investigación, 2020.
Se aplicó el índice kappa y se obtuvo un valor de 0,648, el cual, según Landis y Koch ${ }^{(12)}$, se interpreta como un fuerte acuerdo, por lo que como valor sustancial se puede proceder con la revisión sistemática (Tabla 2).

Tabla 2. Índice kappa

\begin{tabular}{lcc}
\multicolumn{1}{c}{ Índices } & Categoría 1* $^{*}$ & Categoría 2** $^{* *}$ \\
\hline Kappa de la categoría & 0,648 & 0,648 \\
\hline Error kappa de categoría estándar & 0,0641 & 0,0601 \\
\hline IC 95 \% del kappa de la categoría & $\begin{array}{c}\text { Sup.: 0, 5224 } \\
\text { Inf.: 0,7736 }\end{array}$ & $\begin{array}{c}\text { Sup.: 0, } 5302 \\
\text { Inf.: 0,7658 }\end{array}$
\end{tabular}

*Artículos incluidos en el estudio. ${ }^{* *}$ Artículos excluidos del estudio. IC: intervalo de confianza; Inf.: inferior; Sup.: superior. Fuente: datos de la investigación, 2020.

\section{RESULTADOS}

En la Tabla 3 se puede valorar que, en total, se analizaron 19 234 casos de individuos infectados por SARS-CoV-2, ya que el $70 \%(n=21)$ de los artículos fueron realizados en China. Además, cabe mencionar que solo una revista $(3,33 \%)$, de las que publicaron los estudios utilizados en esta revisión sistemática, tiene un factor de impacto inferior a 1,0.

En cuanto a las características de la muestra, existe una mayor prevalencia de hombres, 9943 casos (54,1\%), que de mujeres, 8431 casos (45,9\%). Además, solo 12 (40\%) artículos mencionaron que había comorbilidades hepáticas en un porcentaje de la muestra, y solo 2 (6,7 \%) tenían un porcentaje superior al $9 \%$ de la muestra. Cabe señalar que $3(10 \%)$ artículos tuvieron como muestra solo a pacientes que ingresaron en la unidad de cuidados intensivos (UCI), mientras que $2(6,7 \%)$ artículos analizaron solo casos de muertes por COVID-19 (Tabla 4).

El aumento de AST (4695 casos) fue más prevalente en relación con la cantidad total de casos, cuando se comparó con ALT (3226 casos) (Tabla 5). Solo un estudio no mencionó cambios en los niveles de aminotransferasas ${ }^{(39)}$.

Asghar MS y colaboradores ${ }^{(13)}$ registraron, en una muestra de 100 pacientes, dos casos de insuficiencia hepática fulminante, en aquellos que tenían cambios previos en las enzimas hepáticas. Jiang $S$ y colaboradores ${ }^{(24)}$, en una muestra de 131 pacientes con COVID-19, mencionaron que 76 (58\%) de ellos tenían daño hepático, de los cuales el $81,5 \%$ correspondía a los casos más graves.

Tres estudios mencionaron la presencia de muerte por lesión hepática grave. Jin A y colaboradores ${ }^{(25)}$, con 45 casos, mencionaron que solo un paciente evolucionó con insuficiencia hepática grave (Child-Pugh clase C) y llegó a la muerte; el de Zhang B y colaboradores ${ }^{(38)}$, en el cual, de 
Tabla 3. Características de los estudios seleccionados para revisión

\begin{tabular}{|c|c|c|c|c|}
\hline Artículo & Revista & Tipo de estudio & Local de estudio & Muestra (n) \\
\hline Asghar MS y colaboradores ${ }^{(13)}$ & Cureus Fl: 1,90 & Retrospectivo & Pakistán & 100 \\
\hline Bahl A y colaboradores ${ }^{(14)}$ & Internal and Emergency Medicine (FI: 2322) & Cohorte multicéntrica & Estados Unidos & 1461 \\
\hline Cai $Q$ y colaboradores ${ }^{(15)}$ & Allergy (FI: 8706) & Retrospectivo & China & 298 \\
\hline Chen J y colaboradores ${ }^{(16)}$ & The Journal of Infection (FI: 4603) & Retrospectivo & Shanghái (China) & 249 \\
\hline Chen LY y colaboradores ${ }^{(17)}$ & Journal of Digestive Diseases (FI: 2937) & Retrospectivo & Wuhan (China) & 502 \\
\hline Chen N y colaboradores ${ }^{(18)}$ & Lancet (FI: 60 392) & Retrospectivo & Wuhan (China) & 99 \\
\hline Davies P y colaboradores ${ }^{(19)}$ & The Lancet Child \& Adolescent Health (FI: 8543) & $\begin{array}{l}\text { Observacional } \\
\text { multicéntrico }\end{array}$ & Reino Unido & 78 \\
\hline Du RH y colaboradores ${ }^{(20)}$ & Annals of the American Thoracic Society (FI: 4836) & $\begin{array}{l}\text { Observacional } \\
\text { multicéntrico }\end{array}$ & China & 1017 \\
\hline Falasca L y colaboradores ${ }^{(21)}$ & The Journal of Infectious Diseases (FI: 5186) & Descripción & Italia & 22 \\
\hline Fan Z y colaboradores ${ }^{(22)}$ & Clinical gastroenterology and Hepatology (FI: 8549) & $\begin{array}{l}\text { Retrospectivo de un } \\
\text { solo centro }\end{array}$ & Shanghái (China) & 100 \\
\hline Hu W y colaboradores ${ }^{(23)}$ & Annals of Translational Medicine (FI: 3297) & $\begin{array}{l}\text { Retrospectivo de un } \\
\text { solo centro }\end{array}$ & Wuhan (China) & 16 \\
\hline Jiang S y colaboradores ${ }^{(24)}$ & Frontiers in Medicine (Fl: 3,9) & $\begin{array}{l}\text { Observacional } \\
\text { multicéntrico }\end{array}$ & Zhejiang (China) & 131 \\
\hline Jin A y colaboradores ${ }^{(25)}$ & Biosafety and Health (FI: 1972) & Descriptivo & Pequim (China) & 45 \\
\hline Lei F y colaboradores ${ }^{(26)}$ & Journal of Hepatology AASLD (FI: 14 679) & $\begin{array}{l}\text { Cohorte multicéntrico } \\
\text { retrospectivo }\end{array}$ & China & 5771 \\
\hline Luo S y colaboradores(27) & Clinical Gastroenterology and Hepatology (FI: 8549) & Retrospectivo & Wuhan (China) & 183 \\
\hline Monterde VB y colaboradores ${ }^{(28)}$ & Biomedicines (FI: 4717) & Retrospectivo & Zaragoza (España) & 540 \\
\hline Pan L y colaboradores ${ }^{(29)}$ & $\begin{array}{l}\text { The American Journal of Gastroenterology (FI: } 10 \\
\text { 383) }\end{array}$ & $\begin{array}{l}\text { Descriptivo y } \\
\text { multicéntrico }\end{array}$ & Hubei (China) & 204 \\
\hline $\begin{array}{l}\text { Ramachandran P y } \\
\text { colaboradores }^{(30)}\end{array}$ & $\begin{array}{l}\text { Journal of Clinical and Experimental Hepatology (FI: } \\
\text { 1150) }\end{array}$ & $\begin{array}{l}\text { Retrospectivo de un } \\
\text { solo centro }\end{array}$ & $\begin{array}{l}\text { Nueva York } \\
\text { (Estados Unidos) }\end{array}$ & 145 \\
\hline Richardson S y colaboradores ${ }^{(31)}$ & $\begin{array}{l}\text { Journal of the American Medical Association (FI: } 14 \\
\text { 780) }\end{array}$ & Serie de casos & $\begin{array}{l}\text { Nueva York } \\
\text { (Estados Unidos) }\end{array}$ & 5700 \\
\hline $\begin{array}{l}\text { Schattenberg JM y } \\
\text { colaboradores }^{(32)}\end{array}$ & $\begin{array}{l}\text { United European Gastroenterology Journal (FI: } \\
\text { 3549) }\end{array}$ & Serie de casos & Alemania & 44 \\
\hline Shi M y colaboradores ${ }^{(33)}$ & Journal of Clinical Laboratory Analysis (FI: 1,54$)$ & $\begin{array}{l}\text { Descriptivo } \\
\text { multicéntrico }\end{array}$ & China & 161 \\
\hline Teich VD y colaboradores ${ }^{(34)}$ & Einstein (Fl: 0,261) & $\begin{array}{l}\text { Retrospectivo } \\
\text { unicéntrico }\end{array}$ & Brasil & 510 \\
\hline $\begin{array}{l}\text { Wang Y unicéntricos } \\
\text { colaboradores }^{(35)}\end{array}$ & Journal of Hepatology (FI: 20 582) & Retrospectivo & China & 156 \\
\hline Xie H y colaboradores ${ }^{(36)}$ & Liver International (FI: 5175) & Retrospectivo & China & 79 \\
\hline Yu Y y colaboradores ${ }^{(37)}$ & Critical Care (Fl: 6700) & $\begin{array}{l}\text { Observacional } \\
\text { multicéntrico }\end{array}$ & Wuhan (China) & 226 \\
\hline Zhang B y colaboradores ${ }^{(38)}$ & PLoS One (FI: 2740) & Retrospectivo & China & 82 \\
\hline Zhang G y colaboradores ${ }^{(39)}$ & Respiratory Research (Fl: 3890) & Retrospectivo & China & 95 \\
\hline Zhang S y colaboradores ${ }^{(40)}$ & Critical Care (Fl: 6700) & $\begin{array}{l}\text { Cohorte multicéntrico } \\
\text { retrospectivo }\end{array}$ & China & 828 \\
\hline Zhou F y colaboradores ${ }^{(41)}$ & Lancet (Fl: 60 392) & $\begin{array}{l}\text { Cohorte multicéntrico } \\
\text { retrospectivo }\end{array}$ & Wuhan (China) & 191 \\
\hline Zhou J y colaboradores ${ }^{(42)}$ & Medicine (Baltimore) (FI: 2133) & Descriptivo & Changsha (China) & 201 \\
\hline
\end{tabular}

FI: factor de impacto. Fuente: datos de la investigación, 2020. 
Tabla 4. Características de los estudios seleccionados para revisión

\begin{tabular}{|c|c|c|c|c|c|}
\hline Artículo & Rango de edad (años) & Sexo (M/F) & Comorbilidades hepáticas & $\begin{array}{l}\text { Ingreso a la } \\
\text { UCI }\end{array}$ & Muertes \\
\hline Bahl A y colaboradores ${ }^{(13)}$ & Mediana: 62 (18 a > 80) & $770 / 691$ & - & $374(25,6 \%)$ & $327(22,4 \%)$ \\
\hline Zhou J y colaboradores ${ }^{(14)}$ & Entre 1 y 84 & $102 / 99$ & - & $45(22,4 \%)$ & $2(0,99 \%)$ \\
\hline Jin A y colaboradores ${ }^{(15)}$ & Promedio: 58,8 (entre 7 y 94 ) & $18 / 27$ & - & $9(20 \%)$ & $5(11,1 \%)$ \\
\hline Asghar MS y colaboradores ${ }^{(16)}$ & Promedio: 52,58 & $69 / 31$ & Enfermedad hepática crónica: 9 (9%) & $33(33 \%)$ & $22(22 \%)$ \\
\hline Monterde VB y colaboradores ${ }^{(17)}$ & Promedio: 70 (entre 22 y 99) & $291 / 249$ & - & $61(11,5 \%)$ & $109(20,5 \%)$ \\
\hline Chen LY y colaboradores ${ }^{(18)}$ & Promedio: 61 & $278 / 224$ & - & - & $105(20,9 \%)$ \\
\hline Wang Y y colaboradores ${ }^{(19)}$ & Promedio: 51,2 & $82 / 74$ & - & $9(5,8 \%)$ & $4(2,6 \%)$ \\
\hline Du RH y colaboradores ${ }^{(20)}$ & Promedio: 70,7 & $74 / 35$ & Insuficiencia hepática crónica: 2 (1,8 \%) & $51^{* *}(46,8 \%)$ & $109(100 \%)$ \\
\hline Zhang G y colaboradores ${ }^{(21)}$ & Promedio: 49 & $53 / 42$ & - & $32(33,7 \%)$ & - \\
\hline Chen J y colaboradores ${ }^{(22)}$ & Promedio: 51 & $126 / 123$ & Hepatitis B crónica: 2 (0,8 \%) & $22(8,8 \%)$ & $2(0,8 \%)$ \\
\hline Zhou F y colaboradores ${ }^{(23)}$ & Promedio: 56 (entre 18 y 87) & $119 / 72$ & - & $50(26 \%)$ & $54(28,7 \%)$ \\
\hline Luo S y colaboradores ${ }^{(24)}$ & Promedio: 53,8 & $102 / 81$ & - & - & $7(3,8 \%)$ \\
\hline Pan L y colaboradores ${ }^{(25)}$ & Promedio: 52,9 & $107 / 97$ & - & $16(7,8 \%)$ & $37(17,7 \%)$ \\
\hline Teich VD y colaboradores ${ }^{(26)}$ & Promedio: 39,9 & $290 / 220$ & $\begin{array}{l}\text { Hepatitis B, C, VIH u otra } \\
\text { inmunodeficiencia: } 2(0,4 \%)\end{array}$ & $17(3,4 \%)$ & $1(1,38 \%)$ \\
\hline Lei F y colaboradores ${ }^{(27)}$ & Promedio: 56 & $2724 / 3047$ & Enfermedad hepática crónica: 81 (1,4%) & - & - \\
\hline Chen $\mathrm{N}$ y colaboradores ${ }^{(28)}$ & Promedio: 55,5 & $67 / 32$ & - & - & $11(11,1 \%)$ \\
\hline Yu Y y colaboradores ${ }^{(29)}$ & Promedio: 64 (entre 57 y 70 ) & $139 / 87$ & Hepatopatía crónica: 3 (1,3 \%) & $226(100 \%)$ & $9(4 \%)^{* *}$ \\
\hline Jiang S y colaboradores ${ }^{(30)}$ & Promedio: 51,2 & $70 / 61$ & - & - & - \\
\hline Hu W y colaboradores ${ }^{(31)}$ & Promedio: 44,1 & $6 / 10$ & $\begin{array}{l}\text { Esteatosis: } 3(18,8 \%) \\
\text { Disfunción: } 1(6,3 \%)\end{array}$ & 0 & 0 \\
\hline Zhang S y colaboradores ${ }^{(32)}$ & Promedio: 62 & $447 / 381$ & Enfermedad hepática crónica: 23 (3,02 \%) & $\begin{array}{c}100 \\
(12,08 \%)\end{array}$ & $146(17,63 \%)$ \\
\hline Zhang B y colaboradores ${ }^{(33)}$ & Promedio: 72,5 & $54 / 28$ & Enfermedad hepática: 2 (2,4%) & $14(17,1 \%)$ & $82(100 \%)$ \\
\hline Shi M y colaboradores ${ }^{(34)}$ & Promedio: 59,38 & $104 / 57$ & - & $161(100 \%)$ & $50(31,06 \%)$ \\
\hline $\begin{array}{l}\text { Ramachandran P y } \\
\text { colaboradores }^{(35)}\end{array}$ & Promedio: entre 59,5 y 63 & $55 / 90$ & - & - & $56(38,62 \%)$ \\
\hline $\begin{array}{l}\text { Schattenberg JM y } \\
\text { colaboradores }^{(36)}\end{array}$ & Promedio: 68 (23 y 86) & $30 / 14$ & $\begin{array}{l}\text { Enfermedad hepática preexistente: } 10 \\
(23 \%)\end{array}$ & $13(29,5 \%)$ & $2(4,5 \%)$ \\
\hline Falasca L y colaboradores ${ }^{(37)}$ & Promedio: 27 y 92 & $15 / 7$ & - & - & $22(100 \%)$ \\
\hline Richardson S y colaboradores ${ }^{(38)}$ & Promedio: 63 & $3437 / 2263$ & $\begin{array}{l}\text { Cirrosis: } 19(0,4 \%) \\
\text { Hepatitis B: } 8(0,1 \%) \\
\text { Hepatitis C: } 3(0,1 \%)\end{array}$ & $\begin{array}{c}373(14,2 \%) \\
\text { de } 2634 \\
\text { pacientes }\end{array}$ & $\begin{array}{l}553(21 \%) \\
\text { de } 2634 \\
\text { pacientes }\end{array}$ \\
\hline Davies P y colaboradores ${ }^{(39)}$ & Promedio: 11 & $52 / 26$ & - & $78(100 \%)$ & $2(3 \%)$ \\
\hline Fan Z y colaboradores ${ }^{(40)}$ & Promedio: 50 & $73 / 75$ & - & - & 1 \\
\hline Xie H y colaboradores ${ }^{(41)}$ & Promedio: 60 & $44 / 35$ & - & - & - \\
\hline Cai $Q$ y colaboradores ${ }^{(42)}$ & Promedio 47,5 & $145 / 153$ & $\begin{array}{l}\text { Hígado graso alcohólico: } 5 \text { (1,7 \%) } \\
\text { Hígado graso no alcohólico: } 9 \text { (3\%) } \\
\text { Hepatitis B crónica: } 14(4,7 \%)\end{array}$ & $30(10,1 \%)$ & $3(1 \%)$ \\
\hline
\end{tabular}

Los datos son solo de la muestra que evolucionó con la muerte. ${ }^{* *}$ Todos necesitaban ingreso a la UCI, pero solo había disponibilidad para 51 pacientes. ***Al final del estudio, todavía había 204 pacientes ingresados en la UCI. F: femenino; M: masculino; VIH: virus de la inmunodeficiencia humana. Fuente: datos de la investigación, 2020. 
Tabla 5. Hallazgos sugestivos de lesión hepática en los estudios incluidos en la revisión que consideran el aumento de la actividad enzimática (AST y ALT), proteínas, albúmina y bilirrubina

\begin{tabular}{|c|c|c|c|}
\hline Artículo & AST & ALT & Otros \\
\hline Bahl A y colaboradores ${ }^{(13)}$ & Elevada en 711 casos & Elevada en 434 casos & - \\
\hline Zhou J y colaboradores ${ }^{(14)}$ & Elevada en 31 casos & Elevada en 20 casos & - \\
\hline Jin A y colaboradores ${ }^{(15)}$ & - & - & $\begin{array}{l}\text { Diferentes grados de función hepática anormal en } \\
19(42 \%)\end{array}$ \\
\hline Asghar MS y colaboradores ${ }^{(16)}$ & Elevada en 32 casos & Elevada en 22 casos & $\begin{array}{l}\text { Aumento de la bilirrubina total en } 10 \text { casos } \\
\text { Aumento de GGT en 40/77 }\end{array}$ \\
\hline Monterde VB y colaboradores ${ }^{(17)}$ & 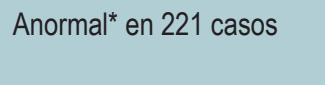 & Elevada em 141 casos & $\begin{array}{l}\text { Prueba hepática anormal en } 319 \text { (64,3\%) } \\
\text { GGT anormal en } 256 \text { casos }\end{array}$ \\
\hline Chen LY y colaboradores ${ }^{(18)}$ & Elevada & & - \\
\hline Wang Y y colaboradores ${ }^{(19)}$ & Elevada & & - \\
\hline Du RH y colaboradores ${ }^{(20)}$ & Elevada en 50 casos & Elevada en 18 casos & - \\
\hline Zhang G y colaboradores ${ }^{(21)}$ & Elevada en 11 casos & Elevada & Disminución de la proteína total en 65 casos \\
\hline Chen J y colaboradores ${ }^{(22)}$ & Elevada & Elevada & Diminución de la albúmina \\
\hline Zhou F y colaboradores ${ }^{(23)}$ & - & Aumentada en 59/189 & - \\
\hline Luo S y colaboradores ${ }^{(24)}$ & $\begin{array}{l}\text { Ligero aumento, promedio: } \\
65,8 \pm 12,7 \mathrm{U} / \mathrm{L}\end{array}$ & $\begin{array}{l}\text { Ligero aumento, promedio: } \\
66,4 \pm 13,2 \mathrm{U} / \mathrm{L}\end{array}$ & - \\
\hline Pan L y colaboradores ${ }^{(25)}$ & Elevada en 22 casos & Elevada en 27 casos & - \\
\hline Teich VD y colaboradores ${ }^{(26)}$ & Elevada en $15 / 70$ & Elevada en $19 / 75$ & Aumento de la bilirrubina total en 3 casos \\
\hline Lei F y colaboradores ${ }^{(27)}$ & Elevada & Aumentada & - \\
\hline Chen $\mathrm{N}$ y colaboradores ${ }^{(28)}$ & Elevada en 35 casos & Elevada en 28 casos & $\begin{array}{l}\text { Disminución de la albúmina en } 97 \text { casos y aumento } \\
\text { de la bilirrubina total en } 18\end{array}$ \\
\hline Yu Y y colaboradores ${ }^{(29)}$ & Elevada en 46 casos & Elevada en 85 casos & $\begin{array}{l}\text { Disminución de la albúmina en } 145 \text { casos y aumento } \\
\text { de la bilirrubina total en } 42\end{array}$ \\
\hline Jiang S y colaboradores ${ }^{(30)}$ & Anormal en 41 casos & Anormal en 45 casos & $\begin{array}{l}\text { Factor de riesgo: tratar con varios fármacos de forma } \\
\text { concomitante }\end{array}$ \\
\hline Hu W y colaboradores ${ }^{(31)}$ & Elevada en 5 casos & Elevada en 5 casos & $\begin{array}{l}\text { Disminución de la albúmina en } 13 \text { casos y aumento } \\
\text { de la bilirrubina total en } 3\end{array}$ \\
\hline Zhang S y colaboradores ${ }^{(32)}$ & - & Elevada & Aumento de la bilirrubina total \\
\hline Zhang B y colaboradores ${ }^{(33)}$ & Elevada en 44 casos & Elevada en 22 casos & $\begin{array}{l}\text { Disminución de la albúmina en } 56 \text { casos y aumento } \\
\text { de la bilirrubina total en } 22\end{array}$ \\
\hline Shi M y colaboradores ${ }^{(34)}$ & Elevada en 82 casos & Anormal en 42 casos & Albúmina anormal en 141 casos \\
\hline Ramachandran P y colaboradores ${ }^{(35)}$ & - & - & Aumento de las aminotransferasas en 20 casos \\
\hline Schattenberg JM y colaboradores ${ }^{(36)}$ & Elevada en 26 casos & Elevada en 6 casos & GGT aumentó en 7 casos \\
\hline Falasca L y colaboradores ${ }^{(37)}$ & - & - & Cambios histopatológicos \\
\hline Richardson S y colaboradores ${ }^{(38)}$ & Elevada en 3263 casos & Elevada en 2176 casos & - \\
\hline Davies P y colaboradores ${ }^{(39)}$ & Sin cambios & Sin cambios & - \\
\hline Fan Z y colaboradores ${ }^{(40)}$ & Elevada en 32 casos & Elevada en 27 casos & $\begin{array}{l}\text { Aumento de GGT en } 26 \text { casos y bilirrubina en } 9 \\
\text { casos }\end{array}$ \\
\hline Xie H y colaboradores ${ }^{(41)}$ & Elevada en 28 casos & Elevada en 25 casos & Aumento de la bilirrubina total en 4 casos \\
\hline Cai $Q$ y colaboradores ${ }^{(42)}$ & Elevada & Elevada & Aumento de GGT \\
\hline
\end{tabular}

Los valores con respecto al tamaño total de la muestra, citados en cada estudio que presentó alteraciones hepáticas, se describen en la Tabla 1. *Anormal: alteración de los valores de referencia. Fuente: datos de la investigación, 2020. 
los 64 pacientes con daño hepático, solo uno evolucionó hasta la muerte; y el estudio de Zhang S y colaboradores ${ }^{(40)}$ con una muestra de 146 muertes, y de este total que murieron, 15 (10,3\%) casos fueron por daño hepático agudo.

Pan L y colaboradores ${ }^{(29)}$, al evaluar los síntomas digestivos y cambios en las enzimas hepáticas (ALT y AST), en una muestra de 204 afectados con COVID-19, observaron que en 49 pacientes que presentaban aumento de ALT y AST, 38 mencionaron síntomas digestivos. Concluyeron que aquellos que tenían síntomas digestivos tenían más probabilidades de tener daño hepatocelular $y$, en consecuencia, cambios enzimáticos. Wang Y y colaboradores ${ }^{(35)}$ mencionaron que el aumento del nivel de estas enzimas estaba relacionado no solo con la gravedad de la enfermedad, sino también con una mayor incidencia de tos.

Zhang G y colaboradores ${ }^{(39)}$ asociaron un aumento de las enzimas ALT y AST, y una reducción del nivel de proteínas totales con un mayor riesgo de ingreso a la UCI, ventilación mecánica y muerte, en los que aproximadamente la mitad de los pacientes presentaba daño de la función hepática. Esta condición también fue citada por Cai Qy colaboradores $^{(15)}$, cuando identificaron a 298 pacientes con COVID19, de los cuales $44(14,8 \%)$ tenían daño hepático, y fue más común en aquellos que manifestaron un cuadro clínico más grave. Sin embargo, no aclararon cuántos de ellos se consideraron casos graves.

Chen LY y colaboradores ${ }^{(17)}$ mencionaron que, de una muestra de 502 individuos, 301 (60\%) pacientes tenían función hepática anormal al ingreso y esta condición se correlacionó con el empeoramiento en la evolución clínica de la infección por SARS-CoV-2; esta progresión también se mencionó en el artículo de Xie $\mathrm{H}$ y colaboradores ${ }^{(36)}$. El empeoramiento de la condición de estos individuos se demostró por una mayor caída en la saturación de oxígeno, un aumento en los recuentos de leucocitos y neutrófilos, niveles más altos de $\mathrm{PCR}$, dímero $\mathrm{D}$, lactato-deshidrogenasa $(\mathrm{LDH})$ y otros. Además, la mortalidad ocurrió en el $28,9 \%$ de los casos de pacientes con función hepática alterada; mientras que en aquellos con función hepática conservada, esta tasa fue del $9 \%{ }^{(17)}$. Este estudio también clasificó la gravedad de la lesión hepática como función hepática normal grado 0 (201 pacientes); grado 1 (138 pacientes) tipo hepatocelular o biliar; y tipo hepatocelular grado 2 (163 pacientes) junto con tipo biliar ${ }^{(17)}$.

$\mathrm{Al}$ analizar la posibilidad de muerte intrahospitalaria, Zhou $\mathrm{F}$ y colaboradores ${ }^{(41)}$ mencionaron que la ALT elevada era uno de los factores para incrementar este riesgo, condición también registrada en otros estudios ${ }^{(14,26)}$. Lei $\mathrm{F}$ y colaboradores ${ }^{(26)}$ consideraron que el riesgo de mortalidad por todas las causas aumentó significativamente 4,81 veces cuando se alteró la AST. Chen J y colaboradores ${ }^{(16)}$ observaron que los niveles bajos de albúmina se encuen- tran entre los factores asociados con el desarrollo del síndrome de malestar respiratorio.

Los autores identificaron cambios histopatológicos en el hígado de pacientes que fallecieron por COVID-19, como infiltrado inflamatorio, congestión y esteatosis ${ }^{(21)}$.

\section{DISCUSIÓN}

En esta revisión sistemática hubo una mayor prevalencia de hombres. En cuanto a las alteraciones de laboratorio de COVID-19, las diferencias entre sexos se acentúan a partir de los 13 años, con cambios en los marcadores de función hepática comunes en diferentes grupos de edad, mientras que la elevación de biomarcadores de inflamación se destaca en hombres mayores ${ }^{(43)}$.

En el escenario de cambios bioquímicos hepatobiliares se observó hiperbilirrubinemia y elevación de aminotransferasas, especialmente de AST, así como elevación de GGT, que muestra daño hepático directo o indirecto por COVID$19^{(44)}$. Entre los mecanismos que pueden justificar cambios en la función hepática por la infección por SARS-CoV-2 está que la liberación exacerbada de citocinas y el consecuente síndrome de dificultad respiratoria aguda (SDRA) promueven la hipoxia y el estrés oxidativo que pueden causar daño hepático ${ }^{(45,46)}$. Además, el receptor ECA-II, utilizado por el SARS-CoV-2 para ingresar a la célula, está presente en las células epiteliales de los hepatocitos y la vía biliar, lo que puede configurar otro mecanismo que justifique la elevación de las enzimas hepáticas observadas y la bilirrubina ${ }^{(4,44)}$.

Con respecto a la asociación entre los cambios en los marcadores hepatocelulares y la gravedad de los pacientes con COVID-19, Shi y colaboradores ${ }^{(47)}$ describieron la AST en niveles normales en pacientes asintomáticos con infección por SARS-CoV-2; mientras que, para pacientes sintomáticos, este marcador estaba elevado, lo que sugiere que los cambios en la función hepática reflejan un peor pronóstico para COVID-19. Entre los otros factores reportados como marcadores de la gravedad de la infección por SARS-CoV-2 se destaca la reducción de los niveles de albúmina, observada en los estudios analizados, y cabe mencionar su relación con la fase inflamatoria aguda ${ }^{(4,44)}$.

El impacto hepático de la COVID-19 en el paciente puede medirse con un análisis histopatológico del hígado, que inicialmente reveló esteatosis microvascular y actividad lobulillar y portal moderada ${ }^{(10,48)}$. En pacientes críticamente enfermos, la biopsia post mortem muestra fibrosis portal, inflamación lobulillar y trombosis vascular significativa ${ }^{(49)}$. Otros hallazgos encontrados en la necropsia son hepatomegalia, infiltración de leucocitos en la zona 1 , necrosis focal en la zona 2 y congestión con trombosis leve en la zona $3^{(50)}$.

En el caso de enfermedad hepática crónica previa a COVID-19, como se observa en algunos de los estudios 
analizados, estos pacientes presentan una activación de la vía alternativa del sistema renina-angiotensina que promueve una reducción de los niveles plasmáticos de angiotensina II y también una elevación de ECA-II ${ }^{(51,52)}$. Sin embargo, en la literatura se describe que, con la entrada de SARS-CoV-2 en la célula, se produce una disminución de la ECA-II y, en consecuencia, un aumento de los niveles plasmáticos de angiotensina II, y el aumento de esta hormona está relacionado con la carga viral y complicaciones sistémicas de COVID$19^{(53,54)}$. Kulkarni y colaboradores plantearon la hipótesis de que los pacientes con enfermedad hepática crónica experimentarían una infección por SARS-CoV-2 con menos complicaciones ${ }^{(44)}$; sin embargo, se necesitan más estudios sobre el tema para hacer tal afirmación.

Debido a la ausencia, hasta el momento, de un tratamiento definido para la infección por el nuevo coronavirus, ya que aún es necesario demostrar la efectividad de lo ya propuesto, el abordaje terapéutico de esta enfermedad pasa por la administración de fármacos que favorezcan el potencial hepatotóxico, como oseltamivir, hidroxicloroquina, paracetamol y acetaminofén ${ }^{(44,55)}$. Por tanto, es evidente la importancia de los estudios sobre esta relación entre el hígado y la COVID-19, para que los profesionales de la salud, teniendo este conocimiento, puedan reducir el riesgo en sus pacientes y de este modo se evite la muerte con daño hepático por COVID-19, como lo afirman Zhang y colaboradores ${ }^{(56)}$ en su estudio.

Algunos factores pueden considerarse limitaciones en este estudio de revisión. Hubo estudios publicados antes del resultado clínico de todos los pacientes, como en Yu Y y colaboradores $^{(37)}$; en consecuencia, no fue posible evaluar completamente el pronóstico. Du RH y colaboradores ${ }^{(20)}$ mencionaron la falta de disponibilidad de una cama de UCI para los pacientes que la necesitaban debido a la sobrecarga de pacientes, lo que puede interferir directamente con el resultado clínico, dado que el déficit en la atención médica es uno de los factores de interferencia.

Además, varios estudios no mencionaron el número de muestra y describieron los hallazgos de manera subjetiva, como en la investigación de Ley F y colaboradores ${ }^{(26)}$ y Zhang $S$ y colaboradores ${ }^{(40)}$, quienes informaron que solo ocurrieron cambios de laboratorio sin identificar el número de muestra de los pacientes estudiados. En cuanto a los objetivos propuestos, es válido informar que, aunque existen datos en la literatura que reportan la hepatotoxicidad de los fármacos utilizados para tratar la COVID-19, la mayoría de los artículos no abordó la condición en sus revisiones.

\section{CONCLUSIÓN}

Es evidente que existe una relación entre la afectación hepática por COVID-19 y su mortalidad, como se describe en los artículos. Sin embargo, aún existe una limitación en la cantidad y, principalmente, en la homogeneidad de los estudios que realizaron esta valoración. Por ello, es fundamental adoptar las enzimas hepáticas como parámetro en la valoración de pacientes con COVID-19 en futuros estudios, ya que, como se explica en esta revisión, existen reportes de la asociación entre su elevación y una mayor tasa de mortalidad, incluso si el mecanismo por el cual esto ocurre no está completamente esclarecido, ya que es una enfermedad muy reciente.

\section{REFERENCIAS}

1. World Health Organization. Rolling updates on coronavirus disease (COVID-19) [internet]. Global Pandemic Network; 2020 [ 9 de septiembre de 2020]. Disponible en: https://www.globalpandemicnetwork.org/covid-19/ international-business-and-global-virtual-teams/rollingupdates-on-coronavirus-disease-covid-19/

2. Yang P, Wang X. COVID-19: a new challenge for human beings. Cell Mol Immunol. 2020 May; 17(5):555-557. https://doi.org/10.1038/s41423-020-0407-x

3. Bai Y, Yao L, Wei T, Tian F, Jin DY, Chen L, Wang M. Presumed Asymptomatic Carrier Transmission of COVID19. JAMA. 2020;323(14):1406-1407. https://doi.org/10.1001/jama.2020.2565

4. Padilla Machaca PM, Cárdenas Ramírez BE, Cabrera Cabrejos MC. Impacto del COVID-19 en las enfermedades hepáticas y la salud pública en el Perú. Rev Gastroenterol Peru. 2020;40(2):162-72.
5. Chan JF, Yuan S, Kok KH, To KK, Chu H, Yang J, Xing F, Liu J, Yip CC, Poon RW, Tsoi HW, Lo SK, Chan KH, Poon VK, Chan WM, Ip JD, Cai JP, Cheng VC, Chen H, Hui CK, Yuen KY. A familial cluster of pneumonia associated with the 2019 novel coronavirus indicating person-toperson transmission: a study of a family cluster. Lancet. 2020;395(10223):514-523. https://doi.org/10.1016/S0140-6736(20)30154-9

6. Guan WJ, Ni ZY, Hu Y, Liang WH, Ou CQ, He JX, Liu L, Shan H, Lei CL, Hui DSC, Du B, Li LJ, Zeng G, Yuen KY, Chen RC, Tang CL, Wang T, Chen PY, Xiang J, Li SY, Wang JL, Liang ZJ, Peng YX, Wei L, Liu Y, Hu YH, Peng P, Wang JM, Liu JY, Chen Z, Li G, Zheng ZJ, Qiu SQ, Luo J, Ye CJ, Zhu SY, Zhong NS; China Medical Treatment Expert Group for Covid-19. Clinical Characteristics of Coronavirus Disease 2019 in China. N Engl J Med. 
2020;382(18):1708-1720.

https://doi.org/10.1056/NEJMoa2002032

7. Bikdeli B, Madhavan MV, Jimenez D, Chuich T, Dreyfus

I, Driggin E, Nigoghossian C, Ageno W, Madjid M,

Guo Y, Tang LV, Hu Y, Giri J, Cushman M, Quéré I,

Dimakakos EP, Gibson CM, Lippi G, Favaloro EJ, Fareed

J, Caprini JA, Tafur AJ, Burton JR, Francese DP, Wang

EY, Falanga A, McLintock C, Hunt BJ, Spyropoulos AC, Barnes GD, Eikelboom JW, Weinberg I, Schulman S, Carrier M, Piazza G, Beckman JA, Steg PG, Stone GW, Rosenkranz S, Goldhaber SZ, Parikh SA, Monreal M, Krumholz HM, Konstantinides SV, Weitz JI, Lip GYH; Global COVID-19 Thrombosis Collaborative Group, Endorsed by the ISTH, NATF, ESVM, and the IUA, Supported by the ESC Working Group on Pulmonary Circulation and Right Ventricular Function. COVID-19 and Thrombotic or Thromboembolic Disease: Implications for Prevention, Antithrombotic Therapy, and Follow-Up: JACC State-of-the-Art Review. J Am Coll Cardiol. 2020 Jun 16;75(23):2950-2973.

https://doi.org/10.1016/j.jacc.2020.04.031

8. Joung JY, Cho JH, Kim YH, Choi SH, Son CG. A literature review for the mechanisms of stress-induced liver injury.

Brain Behav. 2019;9(3):e01235.

https://doi.org/10.1002/brb3.1235

9. Li J, Li RJ, Lv GY, Liu HQ. The mechanisms and strategies to protect from hepatic ischemia-reperfusion injury. Eur Rev Med Pharmacol Sci. 2015;19(11):2036-47.

10. Xu Z, Shi L, Wang Y, Zhang J, Huang L, Zhang C, Liu S, Zhao P, Liu H, Zhu L, Tai Y, Bai C, Gao T, Song J, Xia P, Dong J, Zhao J, Wang FS. Pathological findings of COVID19 associated with acute respiratory distress syndrome. Lancet Respir Med. 2020;8(4):420-422. https://doi.org/10.1016/S2213-2600(20)30076-X

11. Gomes BTL, Souza FAP, Pereira IS, Portela BYM, Santos CRB. Avaliação da função hepática: uma revisão bibliográfica. Mostra Científica de Biomedicina. 2019;4(1).

12. Landis JR, Koch GG. The measurement of observer agreement for categorical data. Biometrics. 1977;33(1):159-74.

13. Asghar MS, Haider Kazmi SJ, Ahmed Khan N, Akram M, Ahmed Khan S, Rasheed U, Hassan M, Memon GM. Correction: Clinical Profiles, Characteristics, and Outcomes of the First 100 Admitted COVID-19 Patients in Pakistan: A Single-Center Retrospective Study in a Tertiary Care Hospital of Karachi. Cureus. 2020;12(8):c34. https://doi.org/10.7759/cureus.c34

14. Bahl A, Van Baalen MN, Ortiz L, Chen NW, Todd C, Milad M, Yang A, Tang J, Nygren M, Qu L. Early predictors of in-hospital mortality in patients with COVID-19 in a large American cohort. Intern Emerg Med. 2020;15(8):1485-1499. https://doi.org/10.1007/s11739-020-02509-7

15. Cai Q, Huang D, Ou P, Yu H, Zhu Z, Xia Z, Su Y, Ma Z, Zhang Y, Li Z, He Q, Liu L, Fu Y, Chen J. COVID-19 in a designated infectious diseases hospital outside Hubei Province, China. Allergy. 2020;75(7):1742-1752. https://doi.org/10.1111/all.14309
16. Chen J, Qi T, Liu L, Ling Y, Qian Z, Li T, Li F, Xu Q, Zhang Y, Xu S, Song Z, Zeng Y, Shen Y, Shi Y, Zhu T, Lu $\mathrm{H}$. Clinical progression of patients with COVID-19 in Shanghai, China. J Infect. 2020;80(5):e1-e6. https://doi.org/10.1016/j.jinf.2020.03.004

17. Chen LY, Chu HK, Bai T, Tu SJ, Wei Y, Li ZL, Hu LL, Zhu R, Zhang L, Han CQ, Xiao L, He Q, Song J, Liu WH, Zhu $\mathrm{Q}$, Chen H, Yang L, Hou XH. Liver damage at admission is an independent prognostic factor for COVID-19. J Dig Dis. 2020;21(9):512-518. https://doi.org/10.1111/1751-2980.12925

18. Chen N, Zhou M, Dong X, Qu J, Gong F, Han Y, Qiu Y, Wang J, Liu Y, Wei Y, Xia J, Yu T, Zhang X, Zhang L. Epidemiological and clinical characteristics of 99 cases of 2019 novel coronavirus pneumonia in Wuhan, China: a descriptive study. Lancet. 2020;395(10223):507-513. https://doi.org/10.1016/S0140-6736(20)30211-7

19. Davies P, Evans C, Kanthimathinathan HK, Lillie J, Brierley J, Waters G, Johnson M, Griffiths B, du Pré P, Mohammad Z, Deep A, Playfor S, Singh D, Inwald D, Jardine M, Ross O, Shetty N, Worrall M, Sinha R, Koul A, Whittaker E, Vyas H, Scholefield BR, Ramnarayan P. Intensive care admissions of children with paediatric inflammatory multisystem syndrome temporally associated with SARSCoV-2 (PIMS-TS) in the UK: a multicentre observational study. Lancet Child Adolesc Health. 2020;4(9):669-677. https://doi.org/10.1016/S2352-4642(20)30215-7

20. Du RH, Liu LM, Yin W, Wang W, Guan LL, Yuan ML, Li YL, Hu Y, Li XY, Sun B, Peng P, Shi HZ. Hospitalization and Critical Care of 109 Decedents with COVID-19 Pneumonia in Wuhan, China. Ann Am Thorac Soc. 2020;17(7):839-846. https://doi.org/10.1513/AnnalsATS.202003-225OC

21. Falasca L, Nardacci R, Colombo D, Lalle E, Di Caro A, Nicastri E, Antinori A, Petrosillo N, Marchioni L, Biava G, D’Offizi G, Palmieri F, Goletti D, Zumla A, Ippolito G, Piacentini M, Del Nonno F. Postmortem Findings in Italian Patients With COVID-19: A Descriptive Full Autopsy Study of Cases With and Without Comorbidities. J Infect Dis. 2020;222(11):1807-1815. https://doi.org/10.1093/infdis/jiaa578

22. Fan Z, Chen L, Li J, Cheng X, Yang J, Tian C, Zhang Y, Huang S, Liu Z, Cheng J. Clinical Features of COVID-19Related Liver Functional Abnormality. Clin Gastroenterol Hepatol. 2020;18(7):1561-1566. https://doi.org/10.1016/j.cgh.2020.04.002

23. Hu W, Chen X, He B, Yuan S, Zhang X, Wu G, Liu J, Xu L, Cha W, Xu M, Sun X, Shen H. Clinical characteristics of 16 patients with COVID-19 infection outside of Wuhan, China: a retrospective, single-center study. Ann Transl Med. 2020;8(10):642. https://doi.org/10.21037/atm-20-3422

24. Jiang S, Wang R, Li L, Hong D, Ru R, Rao Y, Miao J, Chen N, Wu X, Ye Z, Hu Y, Xie M, Zuo M, Lu X, Qiu Y, Liang T. Liver Injury in Critically Ill and Non-critically Ill COVID19 Patients: A Multicenter, Retrospective, Observational 
Study. Front Med (Lausanne). 2020;7:347.

https://doi.org/10.3389/fmed.2020.00347

25. Jin A, Yan B, Hua W, Feng D, Xu B, Liang L, Guo C.

Clinical characteristics of patients diagnosed with COVID-

19 in Beijing. Biosaf Health. 2020;2(2):104-111.

https://doi.org/10.1016/j.bsheal.2020.05.003

26. Lei F, Liu YM, Zhou F, Qin JJ, Zhang P, Zhu L, Zhang XJ, Cai J, Lin L, Ouyang S, Wang X, Yang C, Cheng X, Liu W, Li H, Xie J, Wu B, Luo H, Xiao F, Chen J, Tao L, Cheng G, She ZG, Zhou J, Wang H, Lin J, Luo P, Fu S, Zhou J, Ye P, Xiao B, Mao W, Liu L, Yan Y, Liu L, Chen G, Li H, Huang $X$, Zhang BH, Yuan Y. Longitudinal Association Between Markers of Liver Injury and Mortality in COVID-19 in China. Hepatology. 2020;72(2):389-398.

https://doi.org/10.1002/hep.31301

27. Luo S, Zhang X, Xu H. Don't Overlook Digestive Symptoms in Patients With 2019 Novel Coronavirus Disease (COVID-19). Clin Gastroenterol Hepatol. 2020;18(7):1636-1637. https://doi.org/10.1016/j.cgh.2020.03.043

28. Bernal-Monterde V, Casas-Deza D, Letona-Giménez L, de la Llama-Celis N, Calmarza P, Sierra-Gabarda O, Betoré-Glaria E, Martínez-de Lagos M, Martínez-Barredo L, Espinosa-Pérez M, M Arbones-Mainar J. SARS-CoV-2 Infection Induces a Dual Response in Liver Function Tests: Association with Mortality during Hospitalization. Biomedicines. 2020;8(9):328. https://doi.org/10.3390/biomedicines 8090328

29. Pan L, Mu M, Yang P, Sun Y, Wang R, Yan J, Li P, Hu B, Wang J, Hu C, Jin Y, Niu X, Ping R, Du Y, Li T, Xu G, Hu Q Tu L. Clinical Characteristics of COVID-19 Patients With Digestive Symptoms in Hubei, China: A Descriptive, Cross-Sectional, Multicenter Study. Am J Gastroenterol. 2020;115(5):766-773. https://doi.org/10.14309/ajg.0000000000000620

30. Ramachandran P, Perisetti A, Gajendran M, Chakraborti A, Narh JT, Goyal H. Increased Serum Aminotransferase Activity and Clinical Outcomes in Coronavirus Disease 2019. J Clin Exp Hepatol. 2020;10(6):533-539. https://doi.org/10.1016/j.jceh.2020.06.009

31. Richardson S, Hirsch JS, Narasimhan M, Crawford JM, McGinn T, Davidson KW; the Northwell COVID-19 Research Consortium, Barnaby DP, Becker LB, Chelico JD, Cohen SL, Cookingham J, Coppa K, Diefenbach MA, Dominello AJ, Duer-Hefele J, Falzon L, Gitlin J, Hajizadeh N, Harvin TG, Hirschwerk DA, Kim EJ, Kozel ZM, Marrast LM, Mogavero JN, Osorio GA, Qiu M, Zanos TP. Presenting Characteristics, Comorbidities, and Outcomes Among 5700 Patients Hospitalized With COVID-19 in the New York City Area. JAMA. 2020;323(20):2052-2059. https://doi.org/10.1001/jama.2020.6775

32. Schattenberg JM, Labenz C, Wörns MA, Menge P, Weinmann A, Galle PR, Sprinzl MF. Patterns of liver injury in COVID-19 - a German case series. United European Gastroenterol J. 2020;8(7):814-819. https://doi.org/10.1177/2050640620931657
33. Shi M, Chen L, Yang Y, Zhang J, Xu J, Xu G, Li B, Yin Y. Analysis of clinical features and outcomes of 161 patients with severe and critical COVID-19: A multicenter descriptive study. J Clin Lab Anal. 2020;34(9):e23415. https://doi.org/10.1002/jcla.23415

34. Teich VD, Klajner S, Almeida FAS, Dantas ACB, Laselva CR, Torritesi MG, Canero TR, Berwanger O, Rizzo LV, Reis EP, Cendoroglo Neto M. Epidemiologic and clinical features of patients with COVID-19 in Brazil. Einstein (Sao Paulo). 2020;18:eAO6022. https://doi.org/10.31744/einstein_journal/2020ao6022

35. Wang Y, Liu S, Liu H, Li W, Lin F, Jiang L, Li X, Xu P, Zhang L, Zhao L, Cao Y, Kang J, Yang J, Li L, Liu X, Li Y, Nie R, Mu J, Lu F, Zhao S, Lu J, Zhao J. SARS-CoV-2 infection of the liver directly contributes to hepatic impairment in patients with COVID-19. J Hepatol. 2020;73(4):807-816. https://doi.org/10.1016/j.jhep.2020.05.002

36. Xie H, Zhao J, Lian N, Lin S, Xie Q, Zhuo H. Clinical characteristics of non-ICU hospitalized patients with coronavirus disease 2019 and liver injury: A retrospective study. Liver Int. 2020;40(6):1321-1326. https://doi.org/10.1111/liv.14449

37. Yu Y, Xu D, Fu S, Zhang J, Yang X, Xu L, Xu J, Wu Y, Huang C, Ouyang Y, Yang L, Fang M, Xiao H, Ma J, Zhu W, Hu S, Hu Q, Ding D, Hu M, Zhu G, Xu W, Guo J, Xu J, Yuan H, Zhang B, Yu Z, Chen D, Yuan S, Shang Y. Patients with COVID-19 in 19 ICUs in Wuhan, China: a cross-sectional study. Crit Care. 2020;24(1):219. https://doi.org/10.1186/s13054-020-02939-x

38. Zhang B, Zhou X, Qiu Y, Song Y, Feng F, Feng J, Song Q, Jia Q Wang J. Clinical characteristics of 82 cases of death from COVID-19. PLoS One. 2020;15(7):e0235458. https://doi.org/10.1371/journal.pone.0235458

39. Zhang G, Zhang J, Wang B, Zhu X, Wang Q, Qiu S. Analysis of clinical characteristics and laboratory findings of 95 cases of 2019 novel coronavirus pneumonia in Wuhan, China: a retrospective analysis. Respir Res. 2020;21(1):74. https://doi.org/10.1186/s12931-020-01338-8

40. Zhang S, Guo M, Duan L, Wu F, Hu G, Wang Z, Huang Q, Liao T, Xu J, Ma Y, Lv Z, Xiao W, Zhao Z, Tan X, Meng D, Zhang S, Zhou E, Yin Z, Geng W, Wang X, Zhang J, Chen J, Zhang Y, Jin Y. Development and validation of a risk factor-based system to predict short-term survival in adult hospitalized patients with COVID-19: a multicenter, retrospective, cohort study. Crit Care. 2020;24(1):438. https://doi.org/10.1186/s13054-020-03123-x

41. Zhou F, Yu T, Du R, Fan G, Liu Y, Liu Z, Xiang J, Wang Y, Song B, Gu X, Guan L, Wei Y, Li H, Wu X, Xu J, Tu $\mathrm{S}$, Zhang Y, Chen $\mathrm{H}, \mathrm{Cao}$ B. Clinical course and risk factors for mortality of adult inpatients with COVID-19 in Wuhan, China: a retrospective cohort study. Lancet. 2020;395(10229):1054-1062. https://doi.org/10.1016/S0140-6736(20)30566-3

42. Zhou J, Sun J, Cao Z, Wang W, Huang K, Zheng F, Xie Y, Jiang D, Zhou Z. Epidemiological and clinical features of 201 COVID-19 patients in Changsha city, Hunan, China. 
Medicine (Baltimore). 2020;99(34):e21824.

https://doi.org/10.1097/MD.0000000000021824

43. Ten-Caten F, Gonzalez-Dias P, Castro Í, Ogava RLT, Giddaluru J, Silva JCS, Martins F, Gonçalves ANA, CostaMartins AG, Araujo JD, Viegas AC, Cunha FQ, Farsky S, Bozza FA, Levin AS, Pannaraj PS, de Silva TI, Minoprio P, Pinheiro da Silva F, Andrade BB, Nakaya HI. In-depth analysis of laboratory parameters reveals the interplay between sex, age, and systemic inflammation in individuals with COVID-19. Int J Infect Dis. 2021;105:579-587. https://doi.org/10.1016/j.ijid.2021.03.016

44. Kulkarni AV, Kumar P, Tevethia HV, Premkumar M, Arab JP, Candia R, Talukdar R, Sharma M, Qi X, Rao PN, Reddy DN. Systematic review with meta-analysis: liver manifestations and outcomes in COVID-19. Aliment Pharmacol Ther. 2020;52(4):584-599. https://doi.org/10.1111/apt.15916

45. Mehta P, McAuley DF, Brown M, Sanchez E, Tattersall RS, Manson JJ; HLH Across Speciality Collaboration, UK. COVID-19: consider cytokine storm syndromes and immunosuppression. Lancet. 2020;395(10229):1033-1034. https://doi.org/10.1016/S0140-6736(20)30628-0

46. Hu LL, Wang WJ, Zhu QJ, Yang L. [Novel coronavirus pneumonia-related liver injury: etiological analysis and treatment strategy]. Zhonghua Gan Zang Bing Za Zhi. 2020;28(2):97-99. https://doi.org/10.3760/cma.j.issn.1007-3418.2020.02.001

47. Shi H, Han X, Jiang N, Cao Y, Alwalid O, Gu J, Fan Y, Zheng C. Radiological findings from 81 patients with COVID-19 pneumonia in Wuhan, China: a descriptive study. Lancet Infect Dis. 2020;20(4):425-434. https://doi.org/10.1016/S1473-3099(20)30086-4

48. Qi X, Liu C, Jiang Z, Gu Y, Zhang G, Shao C, Yue H, Chen Z, Ma B, Liu D, Zhang L, Wang J, Xu D, Lei J, Li X, Huang H, Wang Y, Liu H, Yang J, Pan H, Liu W, Wang W, Li F, Zou S, Zhang H, Dong J. Multicenter analysis of clinical characteristics and outcomes in patients with COVID-19 who develop liver injury. J Hepatol. 2020;73(2):455-458. https://doi.org/10.1016/j.jhep.2020.04.010

49. Vaduganathan M, Vardeny O, Michel T, McMurray JJV, Pfeffer MA, Solomon SD. Renin-Angiotensin-Aldosterone System Inhibitors in Patients with Covid-19. N Engl J Med. 2020;382(17):1653-1659.

https://doi.org/10.1056/NEJMsr2005760
50. Liu Y, Yang Y, Zhang C, Huang F, Wang F, Yuan J, Wang Z, Li J, Li J, Feng C, Zhang Z, Wang L, Peng L, Chen L, Qin Y, Zhao D, Tan S, Yin L, Xu J, Zhou C, Jiang C, Liu L. Clinical and biochemical indexes from $2019-\mathrm{nCoV}$ infected patients linked to viral loads and lung injury. Sci China Life Sci. 2020;63(3):364-374. https://doi.org/10.1007/s11427-020-1643-8

51. Sonzogni A, Previtali G, Seghezzi M, Alessio MG, Gianatti A, Licini L, Zerbi P, Carsana L, Rossi R, Lauri E, Pellegrinelli A, Nebuloni M. Liver and COVID 19 infection: a very preliminar lesson learnt from histological post-mortem findings in 48 patients. Preprints. 2020; 2020040438. https://doi.org/10.20944/preprints202004.0438.v1

52. Liu Q, Wang RS, Qu GQ, Wang YY, Liu P, Zhu YZ, Fei G, Ren L, Zhou YW, Liu L. Gross examination report of a COVID-19 death autopsy. Fa Yi Xue Za Zhi. 2020;36(1):21-23. https://doi.org/10.12116/j.issn.1004-5619.2020.01.005

53. Casey S, Schierwagen R, Mak KY, Klein S, Uschner F, Jansen C, Praktiknjo M, Meyer C, Thomas D, Herath C, Jones R, Trebicka J, Angus P. Activation of the Alternate Renin-Angiotensin System Correlates with the Clinical Status in Human Cirrhosis and Corrects Post Liver Transplantation. J Clin Med. 2019;8(4):419. https://doi.org/10.3390/jcm8040419

54. Paizis G, Tikellis C, Cooper ME, Schembri JM, Lew RA, Smith AI, Shaw T, Warner FJ, Zuilli A, Burrell LM, Angus PW. Chronic liver injury in rats and humans upregulates the novel enzyme angiotensin converting enzyme 2 . Gut. 2005;54(12):1790-6. https://doi.org/10.1136/gut.2004.062398

55. Hodgman MJ, Garrard AR. A review of acetaminophen poisoning. Crit Care Clin. 2012;28(4):499-516. https://doi.org/10.1016/j.ccc.2012.07.006

56. Zhang C, Shi L, Wang FS. Liver injury in COVID-19: management and challenges. Lancet Gastroenterol Hepatol. 2020;5(5):428-430. https://doi.org/10.1016/S2468-1253(20)30057-1 